\title{
El compromiso social de la universidad a través de la transferencia de conocimiento en el ámbito de la investigación en Pedagogía Social
}

\author{
Miguel Melendro $\left({ }^{*}\right)$, Ángel De-Juanas $\left({ }^{*}\right)$, Francisco Javier García-Castilla $\left(^{*}\right)$ y Paloma Valdivia $\left({ }^{* *}\right)$ \\ (*) Universidad Nacional de Educación a Distancia (UNED) - Madrid - España (**) Universitat Autónoma de Barcelona - Barcelona - España.
}

\begin{abstract}
RESUMEN
La universidad del nuevo milenio debe ser capaz de producir investigación de calidad, que dé lugar a innovaciones orientadas a mejorar el bienestar de la sociedad de su tiempo. Partiendo de ello, el objetivo del estudio ha sido explorar el grado de compromiso con la sociedad de la investigación universitaria en pedagogía social, a través de un sistema de indicadores sobre procesos activos de transferencia de conocimientos. Se da respuesta a este objetivo a través de un estudio exploratorio de 47 investigaciones, realizadas por 41 grupos de investigación y en las que han participado 131 universidades entre 2013 y 2018. El estudio aporta la construcción de un sistema de indicadores de transferencia de conocimiento en pedagogía social, su aplicación y una primera panorámica de la posición de la pedagogía social en relación con la "tercera misión" de la universidad. Los resultados obtenidos muestran un buen posicionamiento, con investigaciones diversificadas en temáticas, población de referencia y metodologías de investigación; proyectos consistentes y de calidad, trabajados muy frecuentemente en red y desde un prisma interdisciplinar, y sobre todo con impacto académico, pero también un elevado impacto social y profesional. Sin embargo, se detecta una escasa formalización en cuanto al registro de la transferencia social y profesional.
\end{abstract}

Palabras Clave: Transferencia de conocimiento, Investigación, Universidad, Pedagogía social, Indicador.

\section{University's social commitment through knowledge transfer in the research of Social Pedagogy}

\section{ABSTRACT}

A new millennium university must be able to produce high quality research which results in innovation focused on improving the wellbeing of its time's society. On this basis, this research's objective has been to study universities and their degree of commitment with their research on Social Pedagogy. This has been done using an indicators system of knowledge transfer active processes. The answer to this goal has been provided through an exploratory study of 47 researches, done by 41 research teams in which 131 universities have participated between 2013 and 2018. The study provides the construction of a knowledge transfer indicators system in Social Pedagogy, its application and a first panoramic perspective of Social Pedagogy's position in terms of a university's "third mission". The results show a good positioning, with researches diversified on subjects, population and research methodologies. There are consistent and good quality projects, often interdisciplinary and networked, with academic impact and especially with a great social and professional impact, even though there is a lack of formalisation in terms of registering this social and professional transfer.

Keywords: Knowledge transfer, Research, University, Social pedagogy, Social education, Indicator.

\section{Introducción}

Como ha planteado recientemente la Conferencia de Rectores de las Universidades Españolas (CRUE), "la actividad de transferencia de conocimiento y de innovación es uno de los problemas centrales de la economía española y, en consecuencia, de los resultados de las universidades" (Armenteros y García, 2017, p.112). En ese sentido, la universidad española del nuevo milenio no puede limitarse a ser un instrumento de mera transmisión de conocimientos; debe ser capaz de generar esos conocimientos y también de producir una investigación de calidad, que dé lugar a innovaciones orientadas a mejorar el bienestar de la sociedad de su tiempo. Esto supondría priorizar la transferencia del conocimiento como elemento esencial de las políticas de investigación universitaria, intensificando las relaciones y la participación de instituciones públicas, entidades sociales y empresas en los proyectos de investigación, en la línea propuesta desde el Consejo Superior de Investigaciones Científicas (CSIC, 2015): 
"La transferencia de conocimiento, definida como un proceso colaborativo orientado a aplicar el conocimiento generado por la investigación científica en un entorno socioeconómico, es clave para que desde la investigación se pueda generar valor y riqueza, entendidos ambos no solo como conceptos exclusivamente económicos, sino también en un sentido social, cultural y político" (p.2).

En relación con ese sentido social, cultural y político, un punto de partida necesario para nuestro trabajo tiene que ver con la transferencia en las ciencias sociales. Como recoge el citado documento del CSIC, tanto instituciones públicas como entidades sociales y empresas reclaman a la universidad una serie de responsabilidades y compromisos en los espacios sociales, a la vez que le plantean su paradójica ausencia. Estos sectores achacan esta situación a que buena parte de la producción investigadora queda recogida en publicaciones, pero no continúa el camino hacia su puesta en valor en otros ámbitos. Se percibe así que "la actividad de la comunidad científica no responde, en general, a motivaciones sociales o económicas, ni a satisfacer necesidades, ni a solucionar problemas" (CSIC, 2015, p.2) y que los objetivos de los investigadores se orientan sobre todo a difundir sus conocimientos y a conseguir recursos que les permitan continuar con sus investigaciones, más que a gestionar la transferencia de estos a la sociedad de forma más diversificada e impactante.

Los investigadores del ámbito social y de las humanidades, por su parte, plantean la necesidad de instrumentos y criterios adecuados para medir los resultados de transferencia. Pero también que la participación del profesorado universitario en actuaciones de transferencia tenga efectos en su carrera científica y que se supere la rigidez actual de los centros públicos de investigación, cuya estructura organizativa obstaculiza el proceso, restándole agilidad y flexibilidad (CSIC, 2015).

Por lo que respecta a la pedagogía social, en su propia construcción epistemológica se encuentra incorporada la idea de transferencia, como veremos más detalladamente en el apartado siguiente.

\section{Transferencia de conocimientos en educación y en Pedagogía Social}

En el ámbito de las ciencias sociales, la pedagogía social ha tenido un espectacular desarrollo en los últimos decenios. Como señalan Caride, Gradaílle, y Caballo (2015), el proceso de reconstrucción de la Pedagogía Social puede considerarse como uno de los hechos más relevantes en el campo académico y profesional de las ciencias de la educación de finales del pasado siglo, en nuestro país. Desde una amplia y sólida elaboración epistemológica, la Pedagogía Social ha dado origen a carreras profesionales como la de educador social y se ha extendido por los cinco continentes. En la propia concepción de la Pedagogía Social se encuentra incorporada la idea de transferencia. Como plantea Caride (2005), la Pedagogía Social concibe "el quehacer pedagógico-social como una respuesta reflexiva, consciente y estratégica a necesidades, demandas o problemáticas específicas de los ciudadanos... que ha de traducirse en compromisos con valores e ideales considerados fundamentales para conformar una sociedad éticamente habitable... que sea congruente con el desarrollo integral de las personas y su plena realización en la vida cotidiana" (p.97). Un posicionamiento muy en línea con el concepto de Educación social, entendida como acción sistemática para el desarrollo de la sociabilidad y la autonomía del sujeto, buscando su integración y participación crítica que procura la transformación del entorno social en el que habita (Riera, 1996; Pérez Serrano, 2004). Y también con lo que planteara Freire (1999), como rol de quien educa:
"El mundo no es. El mundo está siendo...mi papel en el mundo no es sólo el de quien constata lo que ocurre, sino también el de quien interviene como sujeto de lo que va a ocurrir. No soy un mero objeto de la historia sino, igualmente, su sujeto" (p.75).

Si la transferencia de conocimientos a la sociedad debe ser un objetivo permanente de las universidades en el ámbito de las ciencias sociales, en pedagogía social ésta debe ser una prioridad. De hecho, hay un claro reconocimiento de este papel, y en algún caso de sus implicaciones, si no tanto en la Pedagogía Social en concreto, sí en el campo de la educación. Un reconocimiento multifacético de la transferencia -como el producido en el terreno de la investigación-, pero también como transferencia de conocimientos en relación con los aprendizajes y la formación (Hurtado, Vilà y Sánchez-Martí, 2018; Vilà y Rubio, 2016; Awad, 2015; Dagenais et al., 2016; Feixas y Lagos, 2015; Amador y Hermosilla, 2010). Si bien estos dos últimos tipos de transferencia no aluden al marco conceptual utilizado en este trabajo, sí suponen concreciones metodológicas muy interesantes y aportaciones desde una perspectiva menos académica y más centrada en los procesos de enseñanza-aprendizaje o de formación de agentes educativos. En cuanto a las implicaciones de la transferencia en el campo de la educación, subrayar con Rossi y Rosli, cómo el personal del área educativa de las universidades está relativamente más involucrado que en otras áreas en la realización de consultorías, eventos públicos, cursos, ... en sus actividades de transferencia y "se compromete más directamente con sus grupos de interés utilizando una variedad de interacciones. La 'Educación' parece tener un perfil de compromiso más diversificado que otras áreas" (Rossi \& Rosli, 2015, p.1978).

El compromiso transformador y crítico de la acción socioeducativa es, además, una constante en la actividad científica y profesional de quienes desarrollan su trabajo en este campo de acción-reflexión (Lock, 2017). Como plantea Caride (2015):

"A las voces críticas, que exigen cambios, la pedagogía social, desde hace décadas, viene sumando sus contribuciones científicas y académicas comprometidas con una lectura política y cívica de la educación como una práctica liberadora, en convergencia con los movimientos de renovación educativa, para los que la educación popular es indisociable de la lucha por los derechos humanos, en el tránsito - siempre inconcluso- hacia una sociedad más libre, justa, democrática e igualitaria". (p.93)

Desde estas premisas, el principal objetivo a desarrollar en este trabajo ha sido indagar acerca del grado de compromiso de la investigación universitaria en pedagogía social con la sociedad de su tiempo, a través de procesos activos de transferencia del conocimiento. Para ello, se plantearon como objetivos específicos la construcción y la posterior aplicación, de forma exploratoria, de un sistema de indicadores sobre procesos activos de transferencia de conocimientos en este ámbito. De forma que se pudiera ayudar a estructurar el campo, aportar información para contrastar y enriquecer esa estructura y ofrecer una primera panorámica de la posición de la pedagogía social y la educación social en la "tercera misión" de la universidad.

Hacia un sistema de indicadores sobre procesos activos de transferencia de conocimientos en pedagogía social

Un objetivo inicial de nuestro trabajo es, pues, el de avanzar en la construcción de un sistema de indicadores sobre procesos activos de transferencia de conocimientos en pedagogía social. La literatura sobre esta temática $\mathrm{y}$, de forma más específica, la 
que se refiere a transferencia en ciencias sociales, si bien no es muy extensa sí aporta algunos elementos básicos para la construcción de ese sistema de indicadores.

Un primer elemento que reseñar es el propio interés social que despierta la transferencia de conocimientos. Como plantea el CSIC, "existe un reconocimiento generalizado de que sólo a través de la investigación se pueden enfrentar con posibilidad de éxito los grandes retos sociales y medioambientales a que se enfrentan las sociedades modernas" (CSIC-UIMP, 2015, p.4). Si bien, a pesar de ese interés y de la excelente percepción social de que disfrutan en nuestro país la actividad investigadora y los investigadores en las encuestas de opinión, en la práctica esto no ha supuesto una movilización de la sociedad para potenciarlos. En consecuencia, se identifica con claridad la necesidad de "cargar" de ese valor social a la investigación. En el concreto escenario de la sociedad del conocimiento, la sociedad red en que nos encontramos, esto supone el acceso abierto a los resultados de la investigación y su impacto social, tanto para dar visibilidad y poner en valor el trabajo científico y de investigación que se lleva a cabo, como para utilizar las redes de investigación como una plataforma que recoja las necesidades y propuestas de los ciudadanos (CSIC-UIMP 2015, p.5).

Este interés social por la transferencia, en el caso del ámbito universitario, se focaliza en dos vertientes: la transferencia académica y la transferencia social. Como plantean Bueno y Casini (2010), "la diversidad de universidades supone una dificultad para identificar una serie de indicadores comunes que permitan cuantificar y evaluar la «tercera misión», pero destacan una primera diferenciación, entre aquéllos indicadores que deberían recoger las posibilidades de interacción con la sociedad fuera del ámbito académico, y los que se refieren propiamente a este ámbito. El problema se centraría entonces, para ellos, en seleccionar un número de indicadores reducido, cuya aplicación no fuera difícil ni costosa para las instituciones que decidieran utilizarlos" (p.50).

Esta necesidad de concretar indicadores eficaces para valorar el impacto de la transferencia es planteada también por el CSIC (2015), que recomienda:

"Generar nuevos indicadores para cuantificar y medir las acciones de transferencia en Humanidades y Ciencias Sociales. Una vez contrastados, promover su inclusión entre los criterios de calidad en la investigación, de manera que tengan un efecto real en la evaluación de la actividad científica y en los incentivos que el sistema ofrece a los investigadores." (p.5)

En este sentido, avanzamos aquí la construcción de un sistema de indicadores que nos permita acceder a los dos tipos de transferencia, desde las indicaciones de los autores que han trabajado este tema (Lock, 2017; Rossi \& Rosli, 2015; Seppo \& Lilles, 2012; Bueno y Casini, 2010; Jensen, Palangkaraya, \& Webster, 2009; Molas-Gallart, Salter, Patel, Scott, \& Durán, 2002).

En cuanto a los indicadores referidos a la transferencia académica, se dispone de referencias muy elaboradas y bien pautadas, útiles para detectar procesos activos de transferencia de conocimientos en el seno mismo del entorno académico y entre sus actores. Son bien conocidas por profesores e investigadores las exigencias sobre divulgación del conocimiento científico, que la legislación establece y las agencias de evaluación jerarquizan -publicaciones, congresos, movilidades-, junto a otro grupo de elementos institucionales relacionados con aspectos económicos y organizativos, como patentes, registros, licencias de knowhow, creación de spin-off... (Sala \& Lluch, 2016; CRUE, 2015; Bueno y Casini, 2010; Molas-Gallart et al., 2002). En cualquier caso, no profundizaremos aquí en estas cuestiones ya que no son un elemento central de este trabajo.
En cuanto a la transferencia social, agrupa una serie de elementos diferenciados que componen un mosaico sobre el impacto de la investigación que va más allá de los límites de la academia. Este tipo de transferencia ha constituido el objetivo central de nuestro estudio por su escasa presencia en la literatura existente y la enorme importancia que tiene, en concreto, en el ámbito de la pedagogía social.

Uno de esos elementos de la transferencia social de conocimientos, la transferencia profesional, cobra una relevancia considerable en el ámbito socioeducativo por el carácter aplicado de los conocimientos y la presencia permanente de "agentes intermediarios", que promueven el uso del conocimiento generado por la investigación entre las partes interesadas en la educación (Awad, 2015). Algunas de las implicaciones y posibilidades de transferencia se refieren a los espacios de formación de profesionales para la intervención socioeducativa, como la cualificación en competencias y su certificación vinculada a procesos de empleo, la creación de redes de aprendizaje mutuo interprofesional que favorezcan el intercambio de experiencias, o las orientaciones para que el conocimiento científico sea más útil en las prácticas profesionales en educación (Hurtado, Vilà, y Sánchez-Martí, 2018; Vilà y Rubio, 2016; Dagenais et al., 2016; Seppo \& Lilles, 2012; Amador y Hermosilla, 2010; Jensen, Palangkaraya, \& Webster, 2009). Otras tienen que ver con la presencia de los profesionales en el diseño, desarrollo y/o evaluación de la propia investigación, como ya contemplan tradicionalmente los diseños centrados en la investigación colaborativa o de KT (Knowledge Translation) (Awad, 2015; Gervais, Souffez, \& Ziam, 2016; Bueno y Casini, 2010; Jensen, Palangkaraya, \& Webster, 2009). Un tercer espacio relevante es el relacionado con la difusión masiva de resultados de investigación en los canales profesionales a través de publicaciones, encuentros, jornadas... o de la aparición de los investigadores y profesionales en medios de comunicación y medios digitales (Robinson-García, Delgado-López \& Torres-Salinas, 2011; Molas-Gallart et al., 2002). Finalmente, un cuarto elemento estructurante tiene que ver con los ámbitos de acción socioeducativa en Pedagogía Social, que han sido utilizados en la clasificación de las temáticas consultadas en este trabajo (Figura 1). Núñez y Úcar (2010), tras una amplia revisión de la literatura existente, resumen en tres grandes ámbitos la intervención en Pedagogía Social: educación especializada, educación de adultos y animación sociocultural.

La implicación de instituciones públicas y entidades sociales en los procesos de investigación es recogida como indicador de transferencia a través de la asistencia de investigadores como invitados a conferencias no académicas, su participación en reuniones de comités asesores de organizaciones no académicas, o la colaboración conjunta en proyectos de investigación y construcción de redes sociales activas (Jensen, Palangkaraya, \& Webster, 2009; Molas-Gallart \& Castro Martínez, 2007; Bueno y Casini, 2010).

Es importante considerar también las aportaciones entre investigación y empresa que presentan un doble beneficio, "como sucede en cualquier proceso de transferencia de conocimiento basado en la confianza, que es siempre bidireccional: el de poder contar en la empresa con equipos más motivados y con acceso a conocimiento científico de frontera, y con investigadores públicos conocedores de las necesidades de la sociedad, a las que la empresa desea responder, de modo que puedan tenerlas en cuenta durante el desarrollo de su actividad investigadora" (CSIC-UIMP 2015, p.4). Son muy numerosas las aportaciones en este sentido, en un ámbito de la transferencia que se ha desarrollado enormemente (Bueno y Casini, 2010; Iensen, Palangkaraya, \& Webster, 2009; Molas-Gallart et al., 2002), especialmente en relación con las tecnologías o al campo de la 
economía, y que está muy ligado a la innovación y la responsabilidad social de empresa (RSE). Esta última como elemento que liga a la empresa con el ámbito de las ciencias sociales a través de los denominados "beneficios intangibles" de la transferencia:

"fundamentalmente beneficios de tipo reputacional, al proyectar tanto en la sociedad en general, como en los clientes y en los empleados de la empresa en particular, valores corporativos que se derivan de cualidades asociadas a la investigación, como 'el reconocimiento del mérito basado en el esfuerzo y el talento', 'el potencial de la investigación para resolver los problemas sociales' o 'el carácter global, sin fronteras, de la investigación', por citar algunos". (CSIC-UIMP 2015, p.4).

Sintetizando todo lo anteriormente expuesto, en la Figura 1 se incorporan los principales indicadores localizados en la literatura existente, como elementos clave del sistema de indicadores sobre procesos activos de transferencia de conocimientos en Pedagogía Social. A partir de ellos se ha desarrollado el estudio exploratorio que se refleja en el apartado siguiente.

Figura 1. Sistema de indicadores sobre transferencia del conocimiento en pedagogía social. Fuente: Elaboración propia.

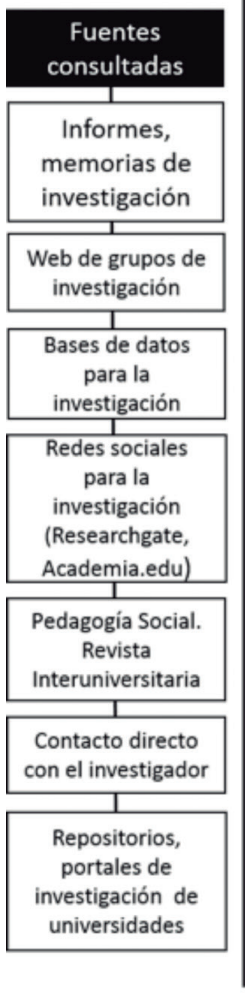

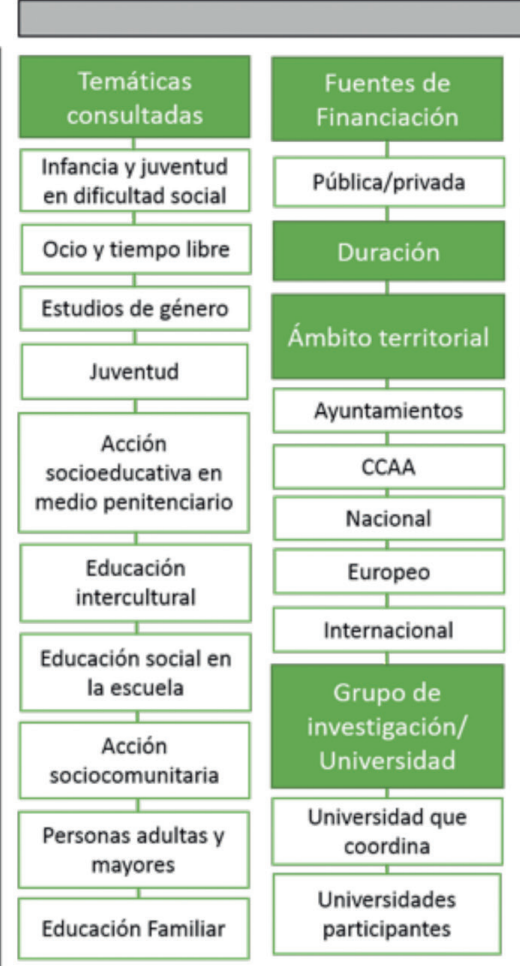

\section{Método}

Para avanzar en el objetivo planteado en este trabajo, se consideró que lo más adecuado era diseñar un estudio exploratorio a partir del análisis de investigaciones relevantes en el campo de la pedagogía social. Se optó por este diseño por considerar que no contábamos con meta-investigaciones sobre el tema, ni con fuentes de información adecuadamente validadas, y era necesario incrementar el conocimiento sobre el fenómeno antes de poder afrontar una investigación más completa. Se trata de un tema nuevo, poco tratado, pero clave para el desarrollo de la considerada como tercera misión de la universidad, el eje de conexión de la universidad con la sociedad de su tiempo: los procesos de transferencia. $\mathrm{O}$ como es definida por Molas-Gallart y Castro-Martínez (2007): “La 'Tercera Misión' se refiere a todas las actividades relacionadas con la generación, uso, aplicación y explotación del conocimiento y otras capacidades universitarias fuera del ámbito académico" (p.1). En un ámbito, el de la pedagogía social, que consideramos clave para identificar necesidades sociales y por la acción socioeducativa que se desarrolla en torno a ellas, tanto dentro como fuera de la enseñanza formal.
Categorias de análisis
Carácter de la

Investigación

¿Es competitiva?

¿Es interdisciplinar?

Tipo de proyecto

Art. 83/contrato

Europeo

Metodología de

investigación

principal

Cuantitativa

Cualitativa

Mixta

Presencia en la

red

e investigación $\mathrm{y} / \mathrm{o}$

especifica de la investigación
Web de grupo/red

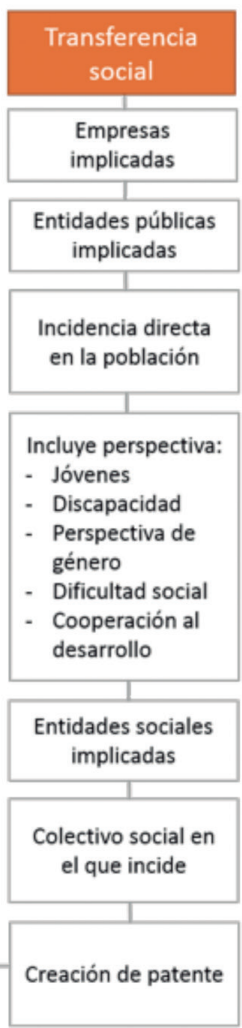

Este estudio ha seguido las indicaciones de Van Teijlingen y Hundley (2001) sobre lo que es un estudio exploratorio: una versión reducida de una investigación más ambiciosa, un estudio de menor escala, flexible, que permite mostrar la diversidad de abordajes teóricos, metodológicos y técnicos posibles, a la vez que evidenciar los problemas que pueden aparecer y la viabilidad y coherencia de los instrumentos y técnicas a utilizar. En cuanto a las limitaciones metodológicas de este tipo de trabajos, coincidiendo con (Hungler y Pólit, 2000), se puede considerar que se trata de un tipo de estudio que no permite establecer una clara concepción explicativa causal y, por tanto, aportar elementos predictivos de lo estudiado.

Se seleccionaron, a partir de estos planteamientos, cuarenta y siete investigaciones desarrolladas en los últimos seis años (2013-2018), que abordan diferentes temáticas pertenecientes al ámbito de la pedagogía social, aunque no exclusivamente y sí en ocasiones compartidas interdisciplinarmente con otros campos de conocimiento. Se partió para ello de una búsqueda sistemática, si bien no se pretendió completar el mapa de la investigación existente en el ámbito de la pedagogía social, sino avanzar en lo que desde la Grounded Theory se plantea como la "saturación" 
del campo de estudio a partir de un muestreo teórico. Es decir, la finalización del muestro cuando la información recopilada no aporta nada nuevo al desarrollo de las propiedades y dimensiones de las categorías de análisis elaboradas (Ardila y Rueda, 2013). El muestreo teórico, por su parte, se ha realizado como muestreo por conveniencia, no probabilístico, a partir de la identificación de investigaciones desde cinco criterios:

1) A partir de los elementos conceptuales básicos que definen el estudio: investigaciones relacionadas con la pedagogía social, la transferencia de conocimientos y su capacidad formativa, de divulgación académica y de impacto social;

2) Desde la selección de las páginas web de los grupos de investigación de una serie de universidades ya estudiadas anteriormente en diferentes trabajos (March, Orte, y Ballester, 2016; Ribas-Machado, 2014) por sus aportaciones al ámbito de la docencia y la investigación en Pedagogía Social.

3) Como plantea la propia idea de transferencia, a partir de los espacios de divulgación a la sociedad del conocimiento adquirido por parte de los investigadores y grupos de investigación. Se partió para ello de los recursos de difusión documentados con que se cuenta en este ámbito del saber; en nuestro país, de forma destacada, se han ocupado de él la Sociedad Iberoamericana de Pedagogía Social y la revista que edita, Pedagogía Social. Revista interuniversitaria. Pero también se ha tenido en cuenta la presencia de este tipo de investigaciones en diferentes medios: publicaciones en revistas académicas y profesionales, conferencias, ponencias y comunicaciones en congresos internacionales o su presencia en los medios de comunicación y páginas web.

4) A partir de indicadores de calidad como la financiación de la investigación, su desarrollo por parte de un grupo de investigación -y no únicamente por investigadores a título personal- y su actualidad (últimos seis años, 20132018).

5) En la búsqueda de saturación teórica, por la que se tomó en cuenta también para la selección de esas investigaciones que trataran diversidad de temáticas, enfoques metodológicos, colectivos de referencia y territorios de procedencia.
A partir de esta selección, se llevó a cabo un proceso de categorización y análisis de contenido de las investigaciones seleccionadas, partiendo del sistema de indicadores construido a partir de la literatura existente sobre el tema y desde un enfoque abierto y coaxial (San Martín, 2014). Se realizó un sencillo estudio descriptivo de tipo cuantitativo que ayudara a contextualizar la información recogida y que supone el soporte básico de los resultados obtenidos, descritos en el apartado siguiente. Todo ello con la intención de responder a los objetivos de investigación planteados.

\section{Resultados}

Las 47 investigaciones analizadas han cumplido con el criterio de saturación del campo de estudio, alcanzando un elevado nivel de distribución temática, territorial, metodológica, de grupos de investigación y universidades, y donde no se consideró relevante añadir nuevas investigaciones, de acuerdo con Flick: "El muestreo e integración de material nuevo se acaba cuando la saturación teórica de una categoría o grupo de casos se ha alcanzado, es decir, cuando no emerge ya nada nuevo" (Flick, 2007, p.79). Lo que se encuentra dentro del carácter exploratorio del trabajo, que busca avanzar en el conocimiento del tema y la construcción teórica sin que los resultados puedan ser generalizables, aunque sí representativos de una realidad emergente, como ya se ha comentado.

Pasamos a continuación a reflejar los resultados más destacados del estudio. Se han ordenado en tres criterios: ámbito de referencia, criterios de calidad y tipo de transferencia.

\section{Resultados por ámbito de referencia}

En la Figura 2 se presentan los proyectos de investigación analizados, un total de 47, en función de la Comunidad Autónoma a la que pertenece la universidad que coordina el proyecto.

Entre todas las investigaciones analizadas, se ha constatado la participación de 131 universidades españolas, europeas y de América Latina; las comunidades autónomas de algunas de ellas no figuran en el gráfico, dado que sus universidades han participado en algún proyecto, pero no lo han coordinado.

Como se puede apreciar, las tres CCAA que más aportan al estudio son Cataluña con 10 investigaciones (21,27\%), Andalucía con $9(19,14 \%)$ y Madrid con $7(14,89 \%)$.

Figura 2. Proyectos analizados por Comunidad Autónoma. Fuente: Elaboración propia.

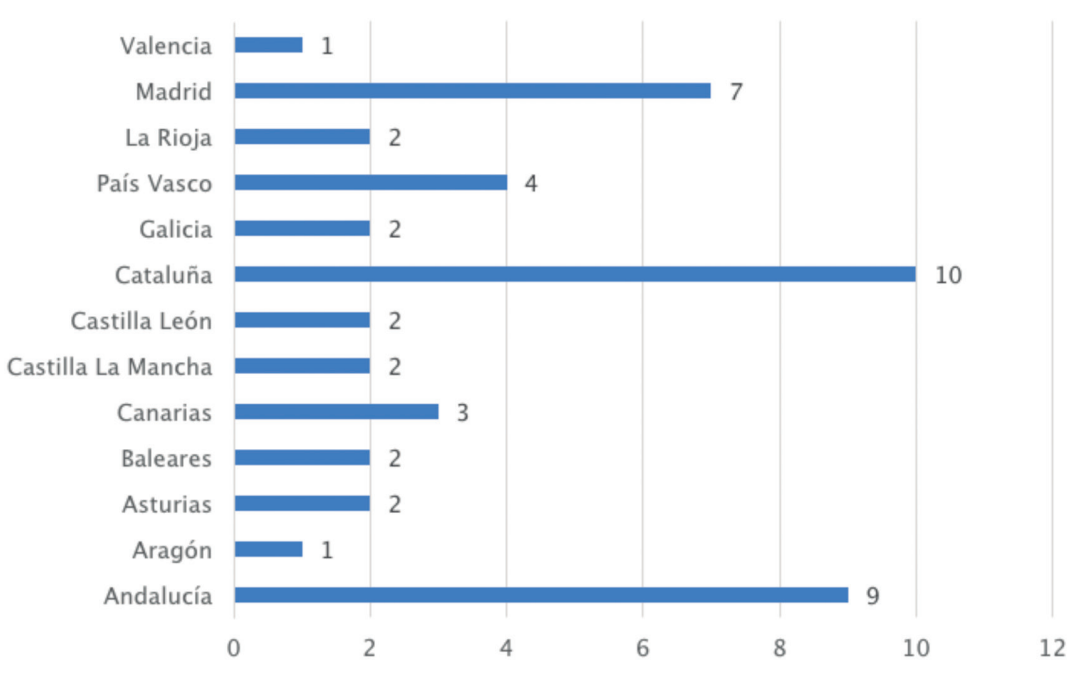

Aula Abierta, volumen 47, no 4, octubre-diciembre, 2018, págs. 403-414 
La mayoría de los proyectos analizados son nacionales (51,06\%); le siguen los proyectos autonómicos (31,91\%), mientras que los proyectos locales, europeos e internacionales representan el 17,03\%, entre todos (Figura 3).

Figura 3. Ámbito territorial. Fuente: Elaboración propia.

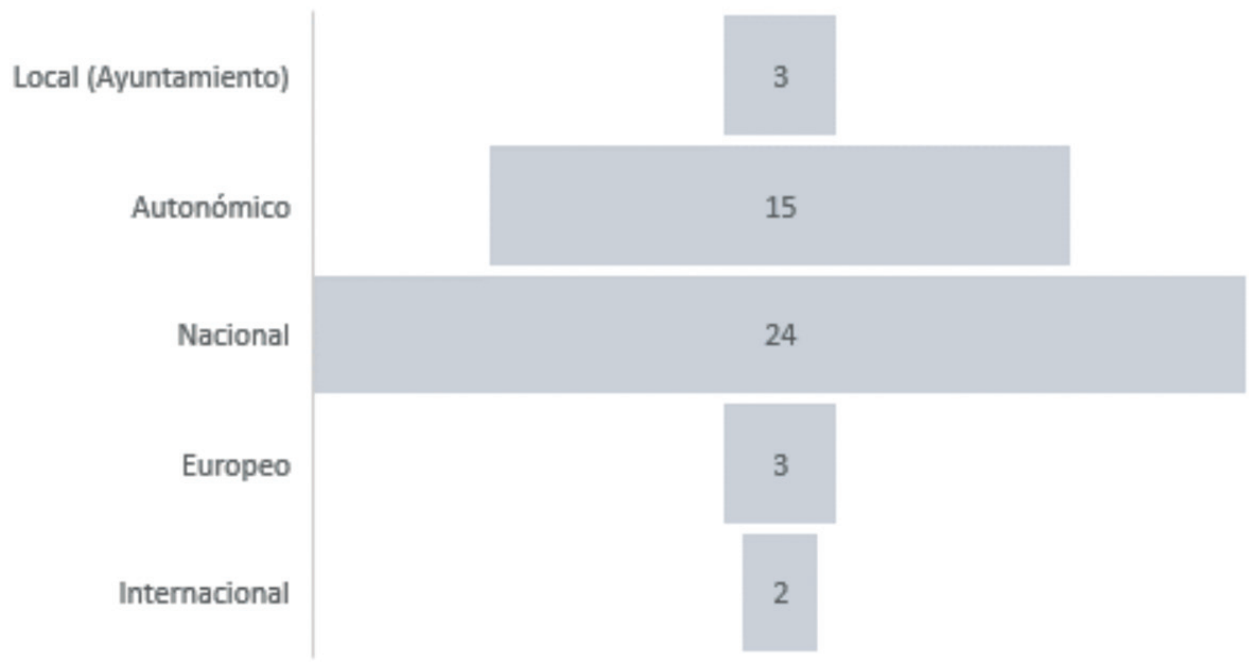

En relación con las temáticas de investigación (Figura 4), por encima de todas destacan las investigaciones sobre la "infancia y juventud en dificultad social", presente en cuatro de cada diez proyectos analizados (42,55\%). Si a ello le sumamos las 6 investigaciones sobre juventud, encontramos que más de la mitad de los proyectos $(55,31 \%)$ se refrieren a población infantil, adolescente o juvenil.
Le siguen, a mayor distancia, las investigaciones sobre Educación Intercultural $(19,15 \%)$, ocio y tiempo libre $(17,03 \%)$ y personas adultas y mayores (14,90\%). Resulta menos frecuente encontrar investigación con transferencia sobre la acción socioeducativa en el medio penitenciario $(4,25 \%)$ y sobre la educación social en la escuela $(6,38 \%)$.

Figura 4. Temáticas de investigación. Fuente: Elaboración propia.

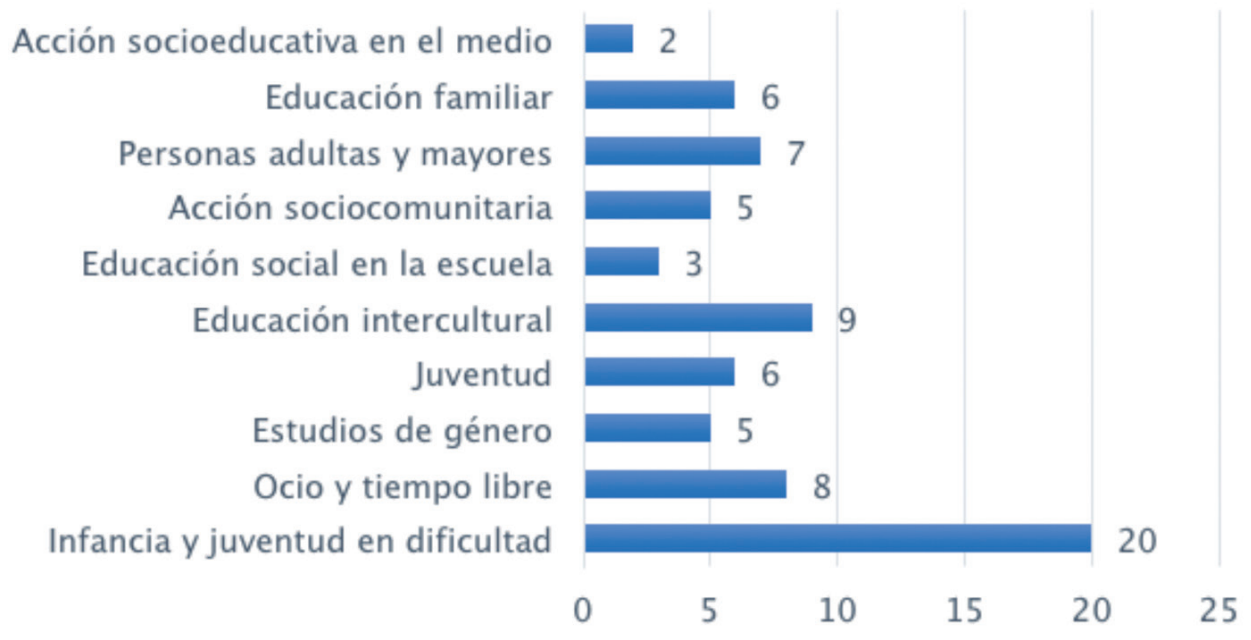

\section{Resultados por criterios de calidad}

Se han consultado investigaciones coordinadas por 41 grupos de investigación, pertenecientes a universidades de 13 de las 17 Comunidades Autónomas. 
Tabla 1. Grupos y redes de investigación

\begin{tabular}{|c|c|c|}
\hline CCAA & Universidad & Grupos y redes de investigación \\
\hline \multirow[t]{9}{*}{ Andalucía } & UPO & Acción Socioeducativa \\
\hline & UGR & Perspectivas feministas en investigación social \\
\hline & & Evaluación de la investigación y de programas educativos andaluces \\
\hline & & Grupo ITACA \\
\hline & UMA & Calidad de vida e intervención comunitaria y organización \\
\hline & & Teoría de la Educación y Educación Social \\
\hline & UHU & ESEIS -Estudios sociales e intervención social- \\
\hline & UAL & Investigación y evaluación en educación intercultural \\
\hline & US & ITACA \\
\hline Aragón & UNIZAR & Grupo EDI -Educación y Diversidad- \\
\hline \multirow[t]{2}{*}{ Asturias } & UNIOVI & IEFES -Intervención Socioeducativa en el ámbito familiar- \\
\hline & & AIES -Análisis, intervención y evaluación socioeducativa- \\
\hline Baleares & UIB & GIFES -Grupo de investigación y formación educativa y social- \\
\hline \multirow[t]{2}{*}{ Canarias } & ULL & $\begin{array}{l}\text { GIOES - Grupo de investigación de Orientación Educativa y Sociolaboral- } \\
\text { GIEI -Grupo de investigación Educación Inclusiva- }\end{array}$ \\
\hline & ULPGC & GIES -Grupo de Investigación en Educación Social- \\
\hline \multirow[t]{2}{*}{ Castilla La Mancha } & UCLM & GIES -Grupo de Investigación en Educación y Sociedad- \\
\hline & & PEC - Grupo de Investigación en Psicología, Educación y Lectura- \\
\hline \multirow[t]{2}{*}{ Castilla y León } & ULE & Grupo de investigación sobre envejecimiento, neurociencia y lógica aplicada \\
\hline & UBU & $\begin{array}{l}\text { FORMADESA -Políticas socioeducativas y económicas para la educación a lo largo de toda la vida, } \\
\text { la promoción de la autonomía de las personas mayores, soportes e-learning y el desarrollo local- }\end{array}$ \\
\hline \multirow[t]{9}{*}{ Cataluña } & UdG & $\begin{array}{l}\text { ERIDIQV - Equipo de Investigación en Infancia, Adolescencia, Derechos de la Infancia y su Cali- } \\
\text { dad de Vida- }\end{array}$ \\
\hline & UB & GRECS - Grup de Recerca sobre Exclusió i Control Socials \\
\hline & & GPS -Grupo de Pedagogía Social- \\
\hline & UOC & LES - Laboratori d’Educació Social \\
\hline & & E-PSICO - Investigadors individuals en Psicologia i Ciències de l’Educació \\
\hline & $\mathrm{UAB}$ & GIPE - Grupo Interdisciplinar de Políticas Educativas- \\
\hline & & IARS -Grup de Recerca en Infància i Adolescència en Risc Social- \\
\hline & UPF & GPS -Grupo de Pedagogía Social- \\
\hline & URL & GIAS -Grupo de Innovación y análisis social- \\
\hline \multirow[t]{2}{*}{ Galicia } & USC & SEPA -Grupo de Investigación Pedagoxía Social Educación Ambiental- \\
\hline & & RESORTES dentro de la Red OCIOGUNE \\
\hline \multirow[t]{2}{*}{ País Vasco } & U. DEUSTO & Ocio y desarrollo Humano \\
\hline & & Intervención: Calidad de Vida e Inclusión Social \\
\hline La Rioja & UR & AFYDO -Actividad física y deporte en el espacio y tiempo de ocio \\
\hline \multirow[t]{6}{*}{ Madrid } & UNED & CISE -Contextos de Intervención Socioeducativa- \\
\hline & & TABA -International Research Group- \\
\hline & & INTER -Investigación en Educación Intercultural- \\
\hline & UAM & GICE -Infancia Contemporánea- \\
\hline & UPM & FCAFYD -Grupo de investigación Psicosocial en el Deporte- \\
\hline & UAH & RILME -Red de investigación sobre liderazgo y mejora de la educación- \\
\hline Valencia & UV & LISIS -Grupo de convivencia escolar- \\
\hline
\end{tabular}

Fuente: Elaboración propia. 
La duración media de los proyectos analizados es de 3 años y medio. El proyecto de menor duración es de un año y medio. La duración de los proyectos más repetida (moda) es de 4 años; hasta 14 proyectos tienen esta duración. Como criterio, se estableció que los proyectos analizados tenían que haberse realizado al menos durante uno de los años del periodo 2013-2018.

En relación con el tipo de investigación (financiada-competitiva), la inmensa mayoría han sido financiados en convocatorias nacionales de proyectos $\mathrm{I}+\mathrm{D}+\mathrm{i}(61,70 \%)$, seguidos de los financiados por contrato a través del artículo 83 de la LOU $(29,78 \%)$ y un pequeño porcentaje de proyectos europeos (6,38\%). En cuanto al carácter competitivo de la investigación, salvo en un caso en el que no se indica y no se encontraron evidencias, en la gran mayoría se trata de investigación competitiva $(89,3 \%)$, mientras que, únicamente, en siete investigaciones encontramos que se trata de investigación no competitiva (8,5\%) (véase Figura 5).

Figura 5. Tipo de investigación. Fuente: Elaboración propia.

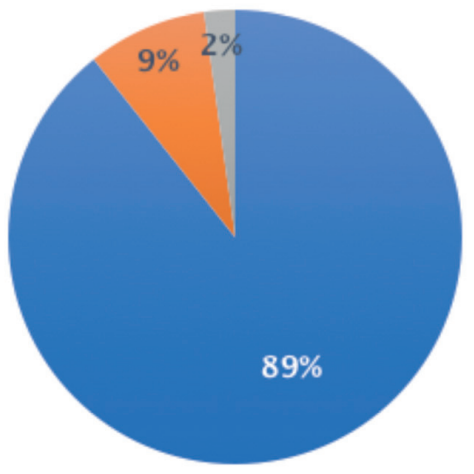

- Competitiva $\quad$ No competitiva No lo indica

En cuanto al tipo de metodología seguida (véase Figura 6), casi en la mitad de los proyectos analizados se ha seguido un tipo de investigación mixta, que integra diferentes tipos metodológicos de investigación (44,68\%). En menor número se encuentran investigaciones sólo de tipo cualitativo (23,40\%); mientras que en último término se encuentran investigaciones de tipo cuantitativo $(12,77 \%)$. Sobre el total de los 47 proyectos analizados, 29 son proyectos de carácter interdisciplinar $(61,70 \%)$.
En relación con la perspectiva de diferentes temas de interés social que se tratan en las investigaciones analizadas, hemos hallado que en 31 ocasiones se ha tenido en consideración la perspectiva de los jóvenes (65,96\%); únicamente en 5 casos se trata la perspectiva de la discapacidad $(10,64 \%)$; en 10 ocasiones se tiene en cuenta la perspectiva de género $(21,27 \%)$; en 19 casos se tiene en cuenta la perspectiva de la dificultad social $(40,42 \%)$; finalmente, solo en dos casos se ha tenido en consideración el tema de la cooperación al desarrollo (4,26\%).

Figura 6. Metodología de investigación. Fuente: Elaboración propia.

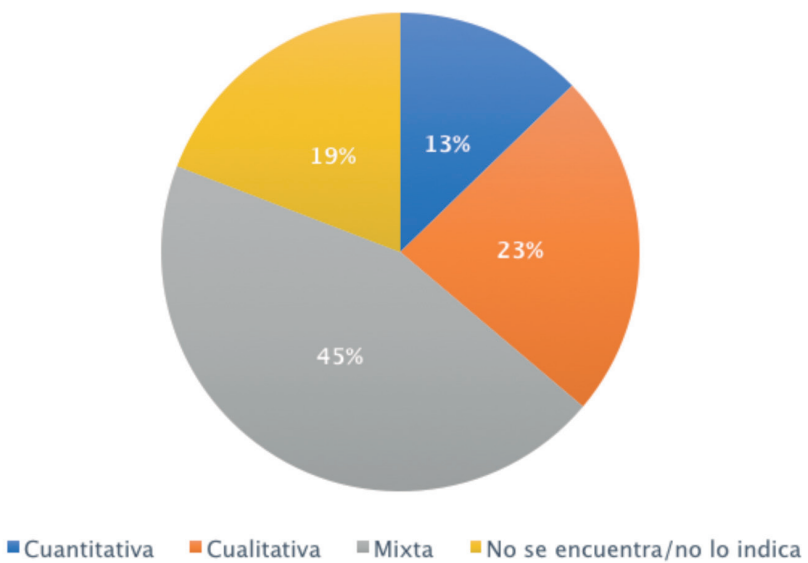

Por último, en cuanto a la difusión de información de grupos y redes a través de páginas web, en la inmensa mayoría de los casos los grupos y redes tienen página web (40 sobre $47,85,11 \%$ ). A su vez, en muchos menos casos los proyectos de investigación se encuentran alojados en una página web propia, siendo algo menos de la mitad de las investigaciones analizadas (23 sobre $47 ; 48,9 \%)$.

\section{Resultados por tipo de transferencia}

El tipo de transferencia que se ha desarrollado en las investigaciones analizadas es el siguiente: 46 proyectos han realizado actividades relacionadas con la transferencia académica $(97,87 \%) ; 25$ con la transferencia profesional $(53,19 \%)$ y 37 con la transferencia social $(78,72 \%)$. Además, un importante número de investigaciones (19), han realizado actividades de transferen- 
cia académica, profesional y social (40,42\%); 15 han desarrollado transferencia académica y social $(31,91 \%)$ y 4 transferencia académica y profesional (8,51\%). Muy pocas investigaciones $(14,89 \%)$ han realizado únicamente transferencia académica.

Figura 7. Tipo de transferencia. Fuente: Elaboración propia.

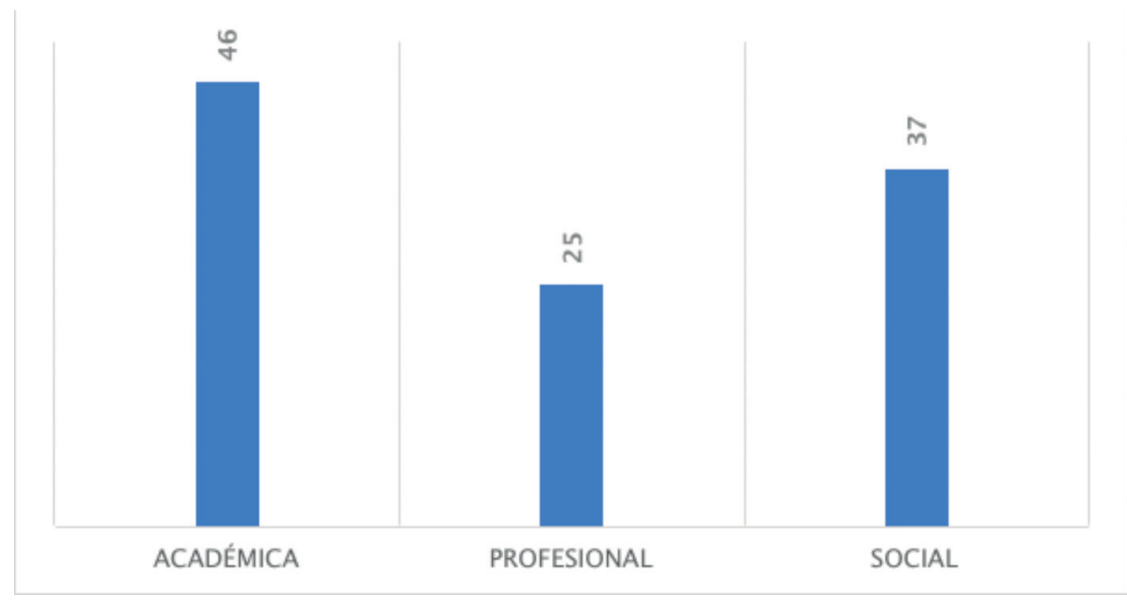

En relación con la mención expresa a la transferencia de investigación en los objetivos de los proyectos analizados, se han encontrado 31 menciones $(66 \%)$.

La transferencia académica se produce prácticamente en todos los proyectos analizados (97,9\% de los casos), mientras que se encuentran evidencias de transferencia social en más de tres cuartas partes de las investigaciones analizadas (78,72\%). Finalmente, en un menor número se encuentran evidencias de transferencia profesional, si bien, esta transferencia concurre con otros tipos en más de la mitad de las investigaciones analizadas $(53,19 \%)$.

De manera concreta, nos centramos en la transferencia académica, en la que hallamos datos interesantes que corroboran que en la gran mayoría de investigaciones analizadas la producción científica está patente. Así, por ejemplo, encontramos que, en 43 de los 47 proyectos analizados, los investigadores han publicado artículos y libros relacionados con la investigación (91,49\%). Asimismo, en 36 casos hemos hallado evidencias sobre participaciones en congresos nacionales e internacionales a modo de ponencias y comunicaciones $(76,60 \%)$. En un menor número, se han hallado cursos, títulos oficiales y propios vinculados con la investigación (25,54\%). También, se ha encontrado cierta transferencia académica vinculada con la elaboración de materiales para la docencia (audiovisuales, materiales didácticos, etc.) (36,17\%). Finalmente, encontramos otros tipos de transferencia académica no por ello menos importantes, como es el caso de tesis dirigidas, trabajos fin de máster y trabajos fin de grado, que han sido resultado directo de las investigaciones analizadas $(46,81 \%)$.
Si nos centramos en la transferencia profesional, hallamos que la participación de los profesionales en el diseño y en la evaluación de las investigaciones analizadas se localiza en 20 casos (42,55\%). Asimismo, se han encontrado publicaciones para profesionales en 15 de las investigaciones examinadas (31,91\%). Igualmente, se han hallado evidencias de la realización de encuentros y jornadas con profesionales en 16 investigaciones (34,04\%). Del mismo modo, se han encontrado evidencias vinculadas a iniciativas destinadas a la formación de profesionales en 10 casos de los 47 analizados (21,27\%). Por último, se han encontrado informes de difusión de la investigación destinados a profesionales en 18 ocasiones $(38,29 \%)$.

En último lugar, cabe tratar la transferencia social de las investigaciones analizadas. En este caso, únicamente hemos encontrado cinco casos en los que haya empresas implicadas (10,64\%); 22 casos sobre 47 en los que hay entidades sociales implicadas (46,80\%); y, finalmente, 25 casos sobre 47 en los que hay instituciones públicas implicadas $(53,20 \%)$. Asimismo, es significativo que sólo hemos encontrado una única patente de registro entre las 47 investigaciones analizadas. También hemos hallado que en 14 investigaciones de las analizadas hay constancia de participación en reuniones de los investigadores con organismos públicos para asesoramiento y toma de decisiones (29,79\%). Finalmente, hemos hallado evidencias de la presencia de estas investigaciones en los medios de comunicación locales, en medios de tirada nacional e incluso en noticiarios de TV y programas de radio. En concreto, en un total de 18 casos de los 47 analizados $(38,29 \%)$. 
Figura 8. Red de universidades por tipo de transferencia ${ }^{1}$. Fuente: Elaboración propia.

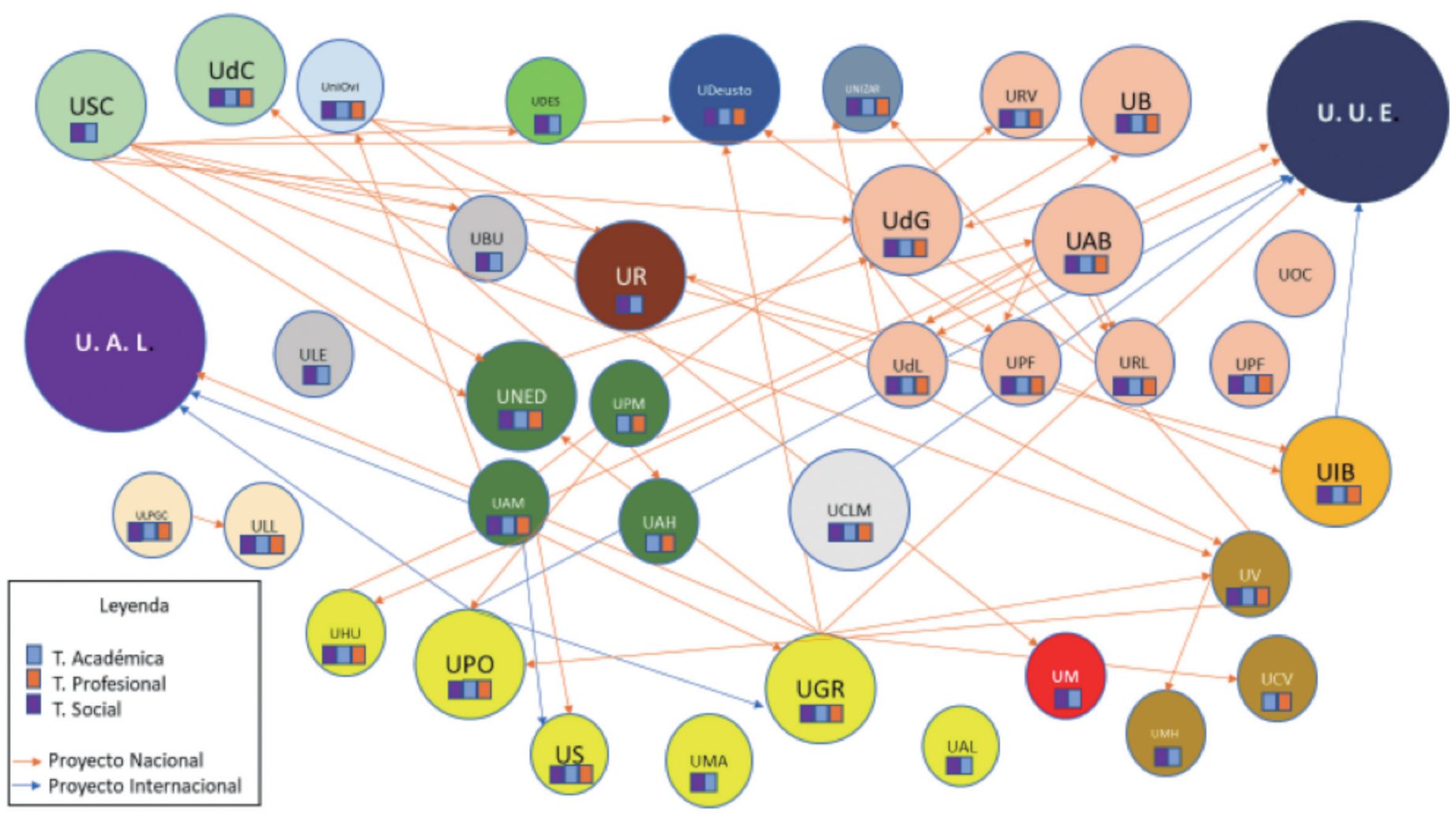

\section{Conclusiones}

De acuerdo con los objetivos de investigación planteados, se describe en este trabajo detalladamente un sistema de indicadores de transferencia en pedagogía social, tomando como referencia la literatura existente sobre el tema. Este sistema ha sido aplicado posteriormente, de acuerdo con el procedimiento planteado, y a partir de esa aplicación se ha podido comprobar tanto su viabilidad como su utilidad para obtener resultados que han aportado información actualizada sobre este campo de estudio. Un campo de estudio sobre el que no se han encontrado otras investigaciones relacionadas con las que contrastar los resultados obtenidos.

Estos resultados muestran cómo la transferencia del conocimiento en pedagogía social incorpora una gran diversidad de temáticas, con un fuerte componente social y educativo, destacando especialmente la referida al grupo de población infantil, adolescente y juvenil. Se aprecia también una gran diversidad de grupos de investigación, muy activos y presentes en la mayoría de las comunidades autónomas, junto a un importante trabajo en red, que llega a movilizar a 131 universidades nacionales e internacionales.

En cuanto a la calidad y el impacto en la sociedad de la transferencia producida, vienen respaldadas por la labor de los grupos de investigación, la permanencia en el tiempo de las investigaciones -que se mantienen activas durante una media de tres años y medio-, el carácter financiado y competitivo de la inmensa mayoría de ellas, al igual que la difusión de conocimientos organizada a través de páginas web. Todo ello ofrece un escenario de consistencia y calidad, que se reflejará en los niveles de transferencia logrados.
Respecto a éstos, aunque predomina, como no podía ser de otro modo, la transferencia académica, la presencia de transferencia social y profesional es muy alta, lo que convierte a estas investigaciones en elementos no solo conocidos, sino también utilizados por la sociedad. Efectivamente, más de la mitad de los proyectos de investigación han desarrollado actividades de transferencia profesional y más de tres cuartas partes de ellos han explorado la vía de la transferencia social, en sus diversas facetas. Esto supone un elevado impacto de los conocimientos transferidos desde la universidad tanto a los colectivos sociales a los que se dirige la pedagogía social, como a las actuaciones de los profesionales que trabajan con ellos fundamentalmente, pero no solo, educadores sociales. Y, a su vez, la generación se nuevos conocimientos a partir de las aportaciones de esos profesionales y colectivos sociales a la universidad. Finalmente, es importante mencionar que un porcentaje muy elevado también, cerca de la mitad de las investigaciones, ha desarrollado los tres tipos de transferencia, académica, profesional y social.

Como hecho destacado, mencionar la facilidad para encontrar -en la mayoría de los casos- información relevante en espacios virtuales, aunque en otros la falta de actualización o la escasa información dificultan la divulgación de los conocimientos acumulados y el acceso a ellos. Otro aspecto que mejorar tiene que ver con el uso muy poco frecuente por parte de los grupos de investigación de recursos estructurales, como la formalización de patentes, registros, creación de spin-off o no hacer explícito el carácter de transferencia de conocimientos de las actividades realizadas o de los propios objetivos de investigación.

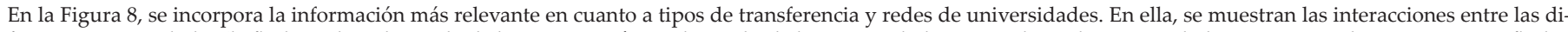

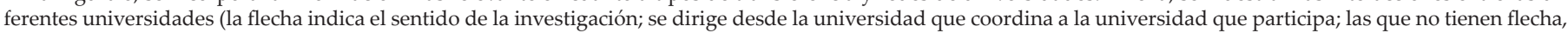

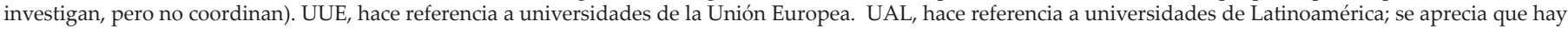
investigaciones nacionales en las que colaboran investigadores de otros países. 
Los resultados obtenidos muestran, por tanto, un buen posicionamiento de la pedagogía social en la transferencia del conocimiento. Coincidiendo con Rossi \& Rosli (2015) en su afirmación de que los investigadores e investigadoras que desarrollan su actividad en el ámbito educativo -en este caso en el de la pedagogía social- están involucrados, en gran medida, en actividades de transferencia. Ponerlo de relevancia y aportar información para estructurar el campo y apoyar la evaluación de su impacto académico, profesional y social ha sido el objetivo de este trabajo, para ofrecer una primera panorámica de la posición de la pedagogía social y la educación social en la "tercera misión" de la universidad.

Todo ello ha venido a reforzar la idea de que la pedagogía social se encuentra comprometida, en una parte importante de su producción científica, con la sociedad de su tiempo.

\section{Referencias}

Amador Muñoz, L. y Hermosilla Rodríguez, J. M. (2010). Generación de conocimiento en la formación de profesionales para la intervención socioeducativa en la infancia. Revista de Humanidades, 17, 191-202.

Ardila Suárez, E. E. y Rueda Arenas, J. F. (2013). La saturación teórica en la teoría fundamentada: su delimitación en el análisis de trayectorias de vida. Revista colombiana de sociología, 36(2), 93-114.

Armenteros, J. H. y García, J. P. (2017). La universidad española en cifras 2015-2016. Madrid: CRUE Universidades españolas.

Awad, N. C. (2015). Knowledge transfer intervention theory: a model grounded in the strategies used by intermediate agents in the context of education. Thèse, Montreal (Canada): Université de Montréal.

Bueno, E. y Casani, F. (2010). La tercera misión de la universidad enfoques e indicadores básicos para su evaluación. Revista de Economía industrial, 366, 43-59.

Caride, J.A. (2005). Las fronteras de la pedagogía social. Perspectivas científica e histórica. Barcelona, España: Gedisa.

Caride, J.A. (2016). La pedagogía social en el diálogo de las universidades con la educación popular y la educación social. Revista Interamericana de Educación de Adultos, 38(1), 85-106.

Caride, J. A., Gradaílle, R., y Caballo, M. B. (2015). De la pedagogía social como educación a la educación social como Pedagogía. Perfiles educativos, 37, 4-11.

CRUE (2015). Investigación y Transferencia de Conocimiento en las universidades españolas 2015. Madrid: Crue Universidades Españolas.

CSIC (noviembre de 2015). Transferencia de conocimiento en humanidades y ciencias sociales. Conclusiones del Encuentro organizado por la Fundación General CSIC. Madrid.

CSIC-UIMP (septiembre de 2015). Responsabilidad social, investigación y empresa. Desarrollo y conclusiones del Encuentro UIMP organizado por la Fundación General CSIC. Santander.

Dagenais, Ch., Pinard, R., St-Pierre, M., Briand-Lamarche, M., Cantave, A. K., \& Peladeau, N. (2016). Using concept mapping to identify conditions that foster knowledge translation from the perspective of school practitioners. Research Evaluation, 25(1), 70-78.

Feixas i Condom, M., Lagos, P., Fernández, I., y Sabaté, S. (2015). Modelos y tendencias en la investigación sobre efectividad, impacto y transferencia de la formación docente en educación superior. Educar, 51(1), 081-107.

Flick, U. (2007). Introducción a la investigación cualitativa. Madrid: Sage.
Freire, P. (1999). Pedagogía da autonomía: saberes necesarios a prática educativa. Sao Paulo, Brasil: Paz e Terra.

Gervais, M-J, Souffez, K., \& Ziam, S. (2016). Quel impact avonsnous ? Vers l'élaboration d'un cadre pour rendre visibles les retombées du transfert des connaissances. Revue francophone de recherche sur le transfert et l'utilisation des connaissances, 1(2), 1-21.

Hungler, B.P. y Pólit, D. (2000). Investigación científica en ciencias de la Salud. México DF, México: Editorial McGrawHill Interamericana.

Hurtado, M. J. R., Baños, R. V., y Martí, A. S. (2018). Una comunidad de práctica virtual para la transferencia del conocimiento entre la universidad y las organizaciones. Revista Tecnología, Ciencia y Educación, 10, 91-108.

Jensen, P., Palangkaraya, A., \& Webster, E. A. (2009). Guide to Metrics on Knowledge Transfer from Universities to Businesses and Industries in Australia. Occasional Paper No. 03/09. Melbourne, Australia: University of Melbourne: Research Institute of Australia.

Lock, D. (2017). Identity construction amongst knowledge transfer staff in English Higher Education Institutions (Doctoral dissertation), London: UCL (University College London).

March, M. X., Orte, C., y Ballester, L. (2016). La pedagogía social en España: de la reconstrucción académica y profesional a la incerteza científica y social. Pedagogía Social. Revista Interuniversitaria, 27, 95-132.

Molas-Gallart, J. \& Castro-Martínez, E. (2007). Ambiguity and conflict in the development of 'Third Mission' indicators. Research Evaluation, 16(4), 321-330.

Molas-Gallart, J., Salter, A., Patel, P., Scott, A., \& Durán, X. (2002). Measuring third stream activities. Brighton (U.K.): SPRU.

Núñez, H. y Úcar, X. (2010). Los ámbitos de intervención de la Pedagogía Social. Recuperado de https://www.academia. edu/1740758/Los_ámbitos_de_intervención_de_la_Pedagogía_Social

Pérez Serrano, G. (2004). Pedagogía Social y Educación Social. Construcción científica e intervención práctica. Madrid: Narcea.

Ribas Machado, E. (2014). O desenvolvimento da Pedagogia Social sob a perspectiva comparada: o estágio atual no Brasil e Espanha (Tesis doctoral). Universidade de São Paulo, Brasil.

Riera, J. (1996). Concepte, formació i professionalització de l'educador social, el treballador social $i$ el pedagog social. Contrast $i$ comparació de llurs rols professionals i intervencions en l'àmbit de la infància (Tesis Doctoral). Universitat Autònoma de Barcelona, Barcelona, España.

Robinson-García, N.; Delgado-López, E. D., \& Torres-Salinas, D (2011). Cómo comunicar y diseminar información científica en Internet para obtener mayor visibilidad e impacto. Aula Abierta, 39(3), 41-50.

Rossi, F. \& Rosli, A. (2015). Indicators of university-industry knowledge transfer performance and their implication for universities: evidence from the United Kingdom. Studies in Higher Education, 40 (10), 1970-1991.

Sala, F. G., \& Lluch, J. O. (2016). Análisis de las publicaciones españolas en la categoría Psychology Educational de la Web of Science durante el periodo 2004-2013. Aula Abierta, 44(1), 46-54.

San Martín Cantero, D. (2014). Teoría fundamentada y Atlas. ti: recursos metodológicos para la investigación educativa. $R e-$ vista electrónica de investigación educativa, 16(1), 104-122.

Seppo, M. \& Lilles, A. (2012). Indicators measuring university-industry cooperation. Discussions on Estonian Economic Policy, 20(1), 204.

Van Teijlingen, E. \& Hundley, V. (2001) The importance of pilot studies. Social Research Update, 35, 1-4. 
Vilà Baños, R. y Rubio Hurtado, M.J. (diciembre de 2016) Una comunidad de práctica virtual para la co-transferencia. La visión de tutores y tutoras de prácticas del grado de Pedagogía. En EDUNOVATIC 2016-I Congreso Virtual internacional de Educación, Innovación y TIC. Libro de actas (pp. 17-27). REDINE. Red de Investigación e Innovación Educativa. 